\title{
Analytical Properties of the Generalized Heat Matrix Polynomials Associated with Fractional Calculus
}

\author{
Mohamed Abdalla ${ }^{1,2}{ }^{1,2}$ and Salah Mahmoud Boulaaras $(\mathbb{D})^{3,4}$ \\ ${ }^{1}$ Department of Mathematics, Faculty of Science, King Khalid University, Abha 61471, Saudi Arabia \\ ${ }^{2}$ Mathematics Department, Faculty of Science, South Valley University, Qena 83523, Egypt \\ ${ }^{3}$ Department of Mathematics, College of Sciences and Arts, ArRas, Qassim University, Saudi Arabia \\ ${ }^{4}$ Laboratory of Fundamental and Applied Mathematics of Oran (LMFAO), University of Oran 1, Oran, 31000 Oran, Algeria
}

Correspondence should be addressed to Salah Mahmoud Boulaaras; s.boularas@qu.edu.sa

Received 29 April 2021; Revised 20 May 2021; Accepted 31 May 2021; Published 9 June 2021

Academic Editor: Santosh Kumar

Copyright ( $\odot 2021$ Mohamed Abdalla and Salah Mahmoud Boulaaras. This is an open access article distributed under the Creative Commons Attribution License, which permits unrestricted use, distribution, and reproduction in any medium, provided the original work is properly cited.

In this paper, we introduce a matrix version of the generalized heat polynomials. Some analytic properties of the generalized heat matrix polynomials are obtained including generating matrix functions, finite sums, and Laplace integral transforms. In addition, further properties are investigated using fractional calculus operators.

\section{Overture}

In the past few decades, matrix versions of the orthogonal polynomials have attracted a lot of research interest due to their close relations and various applications in many areas of mathematics, statistics, physics, and engendering, for example, see [1-11].

The recent advances of fractional order calculus (FOC) are dominated by its multidisciplinary applications. Moreover, special functions of fractional order calculus have many applications in various areas of mathematical analysis, probability theory, control systems, and engineering (see, for example, [12-15]).

Moreover, the development of fractional calculus associated with special matrix functions and polynomials has been investigated by many researchers, for example, the recent works [16-22].

Among these classical polynomials are the heat polynomials (also designated as Temperature polynomials) that are polynomial solutions of the heat equation and also are particularly useful in solving the Cauchy problem (see [2326]). Special functions, such as the confluent hypergeometric function, integral error functions, and Laguerre polynomials, have a close link with the generalized heat polynomials intro- duced [27-29]. Further, the generalized heat polynomials are mainly used to construct an approximate solution of a given problem as a linear combination of the polynomials. Such solution satisfies the governing equation and other equations (cf., e.g., [30-35]).

In our investigation here, we define a generalized heat matrix polynomial $\mathbb{H P}_{m}(T ; \xi, v)$. We then establish certain generating matrix functions, finite sum formulas, Laplace transforms, and fractional calculus operators for these polynomials in Sections 3, 4, 5, and 6, respectively. Further, some interesting special cases and concluding remarks of our main results are pointed out in Section 7.

\section{Preliminaries}

In this section, we give some basic definitions and terminologies; for more details, we can be referred to [36, 37].

Here and through the work, let $\mathbb{C}^{d \times d}$ be the vector space of all the square matrices of order $d \in \mathbb{N}$, ( $\mathbb{N}$ is the set of all positive integers) whose entries are in the set of complex numbers $\mathbb{C}$. For a $E \in \mathbb{C}^{d \times d}$, let $\sigma(E)$ be the set of all eigenvalues of $E$ which is called the spectrum of $E$. We have 
$\mu(E):=\max \{\Re(\xi): \xi \in \sigma(E)\}$ and $\tilde{\mu}(E):=\min \{\Re(z): z \in \sigma(E)\}$,

which imply $\tilde{\mu}(E)=-\mu(-E)$. Here, $\mu(E)$ is called the spectral abscissa of $E$, and the matrix $E$ is said to be positive stable if $\tilde{\mu}(E)>0$. Further, let $I$ and 0 denote the identity and zero matrices corresponding to a square matrix of any order, respectively.

If $E$ is a positive stable matrix in $\mathbb{C}^{d \times d}$, then the gamma matrix function $\Gamma(E)$ is well defined as follows (cf., e.g., [11, 38, 39]):

$$
\Gamma(E)=\int_{0}^{\infty} e^{-z} z^{E-I} d z, z^{E-I}:=\exp ((E-I) \ln z) .
$$

Moreover, if $E$ is a matrix in $\mathbb{C}^{d \times d}$ which gratifies

$$
E+n I \text { is invertible for every integer } n \in \mathbb{N}_{0}=\mathbb{N} \cup\{0\} \text {, }
$$

then $\Gamma(E)$ is invertible, its inverse coincides with $\Gamma^{-1}(E)$. Under condition (3), we can write the following Pochhammer matrix symbol

$(E)_{n}=E(E+I) \cdots(E+(n-1) I)=\Gamma(E+n I) \Gamma^{-1}(E)(n \in \mathbb{N})$.

Let $r, k \in \mathbb{N}_{0}$. Also let $(S)_{r}$ and $(Q)_{k}$ be arrays of $r$ commutative matrices $S_{1}, S_{2}, \cdots, S_{r}$ and $k$ commutative matrices $Q_{1}, Q_{2}, \cdots, Q_{k}$ in $\mathbb{C}^{d \times d}$, respectively, such that $Q_{k}$ $+n I$ are invertible for $1 \leq d \leq k$ and all $n \in \mathbb{N}_{0}$. Then, the generalized hypergeometric matrix function ${ }_{r} \mathbf{F}_{k}\left((S)_{r}\right.$; $\left.(Q)_{k} ; z\right)(z \in \mathbb{C})$ can be defined by (see, e.g., $[11,39]$ )

$$
{ }_{r} \mathbf{F}_{k}\left((S)_{r} ;(Q)_{k} ; z\right)=\sum_{s=0}^{\infty} \prod_{j=1}^{r}\left(S_{j}\right)_{s} \prod_{i=1}^{k}\left[\left(Q_{i}\right)_{s}\right]^{-1} \frac{z^{s}}{s !}
$$

In particular, the hypergeometric matrix function ${ }_{2} \mathbf{F}_{1}$ $(S, P ; C ; z) \equiv \mathbf{F}(S, P ; C ; z)$ is defined by

$$
\mathbf{F}(R, P ; C ; z)=\sum_{s=0}^{\infty}(R)_{s}(P)_{s}(C)_{s}^{-1} \frac{z^{s}}{s !}
$$

for matrices $R, P, C$ in $\mathbb{C}^{d \times d}$ such that $C+n I$ is invertible for all $n \in \mathbb{N}_{0}$. Also, note that for $r=1, k=0$ in (9), we have the Binomial type matrix function ${ }_{1} F_{0}(R ;-; z)$ as follows:

$$
{ }_{1} \mathbf{F}_{0}(R ;-; z)=(1-z)^{-R}=I+R z+\frac{R(R+I) z^{2}}{2 !}+\cdots+\frac{(R)_{n} z^{n}}{n !}+\cdots,|z|<1 .
$$

Let $E$ be a positive stable invertible matrix in $\mathbb{C}^{d \times d}$. Then, the $n^{\text {th }}$ Laguerre matrix polynomial is defined in the form (see, e.g., $[11,40]$ )

$$
\begin{aligned}
\mathbf{L}_{n}^{E}(z) & =\sum_{m=0}^{n} \frac{(-1)^{m} z^{m}}{m !(n-m) !}(S+I)_{n}(E+I)_{m}^{-1} \\
& =\frac{(E+I)_{n}}{n !}{ }_{1} \mathbf{F}_{1}(-n I ; E+I ; z), n \in N_{0} .
\end{aligned}
$$

The Laplace transform of $f(\xi)$ is defined by [7].

$$
\mathscr{L}\{f(\xi) ; \lambda\}=\int_{0}^{\infty} e^{-\lambda \xi} f(\xi) d \xi, \mathfrak{R}(\lambda)>0,
$$

provided that the improper integral exists.

Lemma 1. (see [7]). Let $S$ be a positive stable invertible matrix in $\mathbb{C}^{d \times d}$. Then, we have

$$
\mathscr{L}\left\{\xi^{S} ; \lambda\right\}=\lambda^{-(S+I)} \Gamma(S+I), \operatorname{Re}(\lambda)>0
$$

\section{Generalized Heat Matrix Polynomial and Generating Matrix Functions}

A generalized heat matrix polynomial is defined in (11) below; then, a family of generating matrix functions are proposed, see Theorem 4 and Theorem 8 of this section.

Definition 2. Let $T$ be a positive stable matrix in the complex space $C^{d \times d}$ satisfying the spectral condition (3). Then, we define a generalized heat matrix polynomial of degree $m \in$ $\mathbb{N}_{0}$ in the following explicit form:

$$
\begin{aligned}
\operatorname{HP}_{m}(T ; \xi, v)= & \sum_{s=0}^{m} 2^{2 s}\left(\begin{array}{c}
m \\
s
\end{array}\right) \Gamma\left(T+\left(m+\frac{1}{2}\right) I\right) \Gamma^{-1} \\
& \cdot\left(T+\left(m-s+\frac{1}{2}\right) I\right) \xi^{2 m-2 s} v^{s} \\
= & m !(4 v)^{m} \mathbf{L}_{m}^{T-\frac{1}{2} I}\left(\frac{-\xi^{2}}{4 v}\right) ; v>0,
\end{aligned}
$$

where $\mathbf{L}_{m}^{T}(\xi)$ is the Laguerre matrix polynomial in (8).

Remark 3. Note that

$$
\operatorname{HP}_{m}(T ; \xi, v)=(4 v)^{m}\left(T+\frac{1}{2} I\right)_{m}{ }_{1} \mathbf{F}_{1}\left(-m I, T+\frac{1}{2} I ; \frac{-\xi^{2}}{4 v}\right)
$$

and that for the scalar case $d=1$, taking $T=a$ and $a>0$, the $m^{\text {th }}$ polynomial $\operatorname{HP}_{m}(a ; \xi, v)$ coincides with the classical scalar generalized heat polynomial, see [24, 26, 33]. Further, the ordinary heat polynomial defined in [23], when $T=0$; $\operatorname{HP}_{m}(0 ; \xi, v)=v_{2 m}(\xi, v)$.

Theorem 4. Let $\xi \in \mathbb{C}, v>0, m \in \mathbb{N}_{0},\left|\xi^{2} t\right|<|1-4 v t|$ and $T$ and $R$ be positive stable matrices in $C^{d \times d}$ such that $T+n I$ is 
invertible for all $n \in \mathbb{N}_{0}$. A generating matrix function of $\mathbb{H}$ $\mathbb{P}_{m}(T ; \xi, v)$ is

$$
\begin{gathered}
\sum_{m=0}^{\infty}\left[\left(T+\frac{1}{2} I\right)_{m}\right]^{-1}(R)_{m} \mathbb{H P}_{m}(T ; \xi, v) \frac{t^{m}}{m !} \\
=(1-4 v t)^{-R}{ }_{1} \mathbf{F}_{1}\left(R, T+\frac{1}{2} I ; \frac{\xi^{2} t}{1-4 v t}\right) .
\end{gathered}
$$

Proof. For convenience, suppose that the left-hand side of (13) is denoted by J. According to the series expression of (11) and (7) to J, we find that

$$
\begin{aligned}
\mathbf{J} & =\sum_{m=0}^{\infty}\left[\left(T+\frac{1}{2} I\right)_{m}\right]^{-1}(R)_{m} \mathbb{H P}_{m}(T ; \xi, v) \frac{t^{m}}{m !} \\
& =\sum_{m=0}^{\infty} \sum_{s=0}^{m} \frac{(R)_{m}\left[(T+1 / 2 I)_{m-s}\right]^{-1}}{s !(m-k) !}(\xi)^{2 m-2 s}(4 v)^{s} t^{m} \\
& =\sum_{m=0}^{\infty}(R)_{m}\left[\left(T+\frac{1}{2} I\right)_{m}\right]^{-1} \frac{\left(\xi^{2} t\right)^{m}}{m !} \cdot \sum_{s=0}^{\infty}(R+m I)_{s} \frac{(\xi v t)^{s}}{s !} \\
& =(1-4 v t)^{-R} \sum_{m=0}^{\infty}(R)_{m}\left[\left(T+\frac{1}{2} I\right)_{m}\right]^{-1} \frac{\left(\xi^{2} t / 1-4 v t\right)^{m}}{m !}
\end{aligned}
$$

Upon using the relation (6), the last equality evidently leads us to the required result.

Corollary 5. For $\mathbb{H P}_{m}(T ; \xi, v)$, the following generating matrix function holds true

$$
\begin{gathered}
\sum_{m=0}^{\infty}\left[\left(T+\frac{1}{2} I\right)_{m}\right]^{-1} \operatorname{HP}_{m}(T ; \xi, v) \frac{t^{m}}{m !} \\
=\exp (4 v t)_{0} \mathbf{F}_{1}\left(-, T+\frac{1}{2} I ; \xi^{2} t\right) .
\end{gathered}
$$

Remark 6. The Bessel matrix function $J_{R}(z)$, for a positive stable matrix $R \in C^{d \times d}$, is expressible in terms of hypergeometric matrix function as follows (see, e.g., $[11,41]$ )

$$
J_{R}(w)=\left(\frac{w}{2}\right)^{R} \Gamma^{-1}(R+I)_{0} \mathbf{F}_{1}\left(-; R+I, \frac{-w^{2}}{4}\right) .
$$

Thus, by applying the relation (16) to (15) in Corollary 5, we can deduce the following Corollary.

Corollary 7. For $\mathbb{H P}_{m}(T ; \xi, v)$, the following holds true

$$
\begin{aligned}
& \sum_{m=0}^{\infty} {\left[\left(T+\frac{1}{2} I\right)_{m}\right]^{-1} \operatorname{HP}_{m}(T ; \xi, v) \frac{t^{m}}{m !}=\Gamma\left(\left(T+\frac{1}{2} I\right)\right) } \\
& \cdot(i \xi \sqrt{t})^{-\left(T-\frac{1}{2} I\right)} \exp (4 v t) J_{T-\frac{1}{2} I}(2 i \xi \sqrt{t}) .
\end{aligned}
$$

Theorem 8. Let $\xi \in \mathbb{C}, v>0, m, l \in \mathbb{N}_{0}$ and $T$ be a positive stable matrix in $C^{d \times d}$ such that $T+n I$ is invertible for all $n \in \mathbb{N}_{0}$. The following relation holds true

$$
\begin{aligned}
\sum_{m=0}^{\infty} \operatorname{HP}_{m+l}(T ; \xi, v) \frac{t^{m}}{m !}= & (1-4 v t)^{-\left(T+\left(l+\frac{1}{2}\right) I\right)} \exp \\
& \cdot\left(\frac{\xi^{2} t}{1-4 v t}\right) \operatorname{HP}_{m+l}\left(T ; \frac{\xi}{\sqrt{1-4 v t}}, v\right) .
\end{aligned}
$$

Proof. Follows by induction or by the successive application of Theorem 4 when $R=(T+1 / 2 I)$. The details are omitted.

Corollary 9. For $l=0$ in Theorem 8 , the following holds true

$$
\sum_{m=0}^{\infty} \operatorname{HP}_{m}(T ; \xi, v) \frac{t^{m}}{m !}=(1-4 v t)^{-\left(T+\frac{1}{2} I\right)} \exp \left(\frac{\xi^{2} t}{1-4 v t}\right) \text {. }
$$

Remark 10. The special cases of (18) and (19) when $d=1$ are seen to yield the classical generating functions of the generalized Heat polynomials (see [24, 33]).

\section{Finite Sums}

Here, various finite sums of $\mathbb{H P}_{m}(T ; \xi, v)$ can be obtained in the following results.

Theorem 11. Let $\xi, z \in \mathbb{C}, v>0, m \in \mathbb{N}_{0}$, and $T$ be a positive stable matrix in $C^{d \times d}$ such that $T+n I$ is invertible for all $n$ $\in \mathbb{N}_{0}$. Then, we have

$$
\begin{aligned}
\operatorname{HP}_{m}(T ; \xi z, v)= & \sum_{s=0}^{m}\left(\begin{array}{l}
n \\
k
\end{array}\right)\left(T+\frac{1}{2} I\right)_{m}\left[\left(T+\frac{1}{2} I\right)_{s}\right]^{-1} \\
& \cdot\left\{4 v\left(1-z^{2}\right)\right\}^{m-s} z^{2 s} \operatorname{HP}_{s}(T ; \xi z, v) .
\end{aligned}
$$

Proof. From (15) and the following fact

$$
e_{0}^{4 v t} \mathbf{F}_{1}\left(; T+\frac{1}{2} I ; \xi^{2} z^{2} t\right)=e^{4 v t\left(1-z^{2}\right)+4 v z^{2} t}{ }_{0} \mathbf{F}_{1}\left(; T+\frac{1}{2} I ; \xi^{2} z^{2} t\right) .
$$

We thus find that

$$
\begin{aligned}
\sum_{m=0}^{\infty} & \sum_{s=0}^{\infty} \mathbb{H P}_{m}(T ; \xi, v)\left[\left(T+\frac{1}{2} I\right)_{s}\right]^{-1} \frac{\left\{4 v\left(1-z^{2}\right)\right\}^{m}\left(z^{2} t\right)^{s}}{m ! s !} \\
& =\sum_{m=0}^{\infty} \sum_{s=0}^{m} \frac{\left\{4 v\left(1-z^{2}\right)\right\}^{m-s}\left(z^{2} t\right)^{s}}{(m-s) ! s !}\left[\left(T+\frac{1}{2} I\right)_{s}\right]^{-1} \mathbb{H P}_{m}(T ; \xi, v)
\end{aligned}
$$

Comparing the coefficient of $t^{m}$ on both side, we thus get the required a finite sum formula (20). 
Theorem 12. Let $\xi \in \mathbb{C}, v>0, m \in \mathbb{N}_{0}$, also let $T$ and $R$ be positive stable matrices in $C^{d \times d}$ such that $T+n I$ and $R+n I$ are invertible for all $n \in \mathbb{N}_{0}$. Then, we have

$$
\begin{aligned}
\operatorname{HP}_{m}(T ; \xi, v)= & \left(T+\frac{1}{2} I\right)_{m}\left[(R)_{m}\right]^{-1} \sum_{s=0}^{m}\left(\begin{array}{l}
n \\
s
\end{array}\right)\left(T-R+\frac{1}{2} I\right)_{s} \\
& \cdot\left[\left(T+\frac{1}{2} I\right)_{s}\right]^{-1} \times \operatorname{HP}_{s}(T ; i \xi, v) \mathbb{H P}_{m-s} \\
& \cdot(2 R-T-I ; \xi, v) .
\end{aligned}
$$

Proof. By using the series (11) and Theorem 4 with applying to Kummer's matrix formula (see [5]), we observe that

$$
\begin{aligned}
\sum_{m=0}^{\infty}[ & \left.\left(T+\frac{1}{2} I\right)_{m}\right]^{-1}(R)_{m} \mathbb{H P}_{m}(T ; \xi, v) \frac{t^{m}}{m !} \\
= & (1-4 v t)^{-R} \exp \left(\frac{\xi^{2} t}{1-4 v t}\right)_{1} \mathbf{F}_{1}\left(T-R+\frac{1}{2} I, T+\frac{1}{2} I ; \frac{-\xi^{2} t}{1-4 v t}\right) \\
= & (1-4 v t)^{-(2 R-T-I)+\frac{1}{2} I} \exp \left(\frac{\xi^{2} t}{1-4 v t}\right)(1-4 v t)^{-\left(T-R+\frac{1}{2} I\right)_{1} \mathbf{F}_{1}} \\
& \cdot\left(T-R+\frac{1}{2} I, T+\frac{1}{2} I ; \frac{-\xi^{2} t}{1-4 v t}\right) \\
= & \sum_{m=0}^{\infty} \frac{\mathbb{H P}_{m}(2 R-T-I ; \xi, v)}{m !} t^{m} \sum_{s=0}^{\infty}\left(T-R+\frac{1}{2} I\right)_{s} \\
& \cdot\left[\left(T+\frac{1}{2} I\right)_{s}\right]^{-1} \frac{\mathbb{H P}_{m}(T ; i \xi, v)}{m !} t^{s} .
\end{aligned}
$$

Equating the coefficient of $t^{m}$ on both sides, we thus arrive at the desired result (23).

\section{Laplace Transforms}

Here, Laplace integral transforms of the generalized heat matrix polynomials are derived as follows.

Theorem 13. Let $\xi \in \mathbb{C}, v>0, \mathfrak{R}(\lambda)>0, m \in \mathbb{N}_{0}$. Also, let $T$ and $A$ be a positive stable matrices in $C^{d \times d}$ such that $T+n I$ is invertible for all $n \in \mathbb{N}_{0}$. The following Laplace transform formula hold

$$
\begin{aligned}
\mathscr{L}\left\{u^{A} \mathbb{H P}_{m}(T ; \xi \sqrt{u}, v): \lambda\right\}= & (4 v)^{m}\left(T+\frac{1}{2} I\right)_{m} \lambda^{-(A+I)} \Gamma \\
& \cdot(A+I)_{2} \mathbf{F}_{1}\left(-m I, A+I ; T+\frac{1}{2} I ; \frac{-\xi^{2}}{4 v \lambda}\right) .
\end{aligned}
$$

Proof. Making a particular use of (9) with (11), (6) and applying to Lemma 1, yields our desired result (25) in Theorem 13. The details are omitted.

A similar procedure yields the following Laplace integral transforms. So we prefer to omit the proofs.
Theorem 14. Let $\xi \in \mathbb{C}, v>0, \mathfrak{R}(\lambda)>0, m \in \mathbb{N}_{0}$. Also, let $T$ and $A$ be a positive stable matrices in $C^{d \times d}$ such that $T+n I$ is invertible for all $n \in \mathbb{N}_{0}$. The following Laplace transform formula hold

$$
\begin{aligned}
\mathscr{L}\left\{u^{A} \operatorname{HP}_{m}(T ; \xi u, v): \lambda\right\} \\
=(4 v)^{m}\left(T+\frac{1}{2} I\right)_{m} \lambda^{-(A+I)} \Gamma(A+I)_{3} \mathbf{F}_{1} \\
\\
\quad\left(-m I, \frac{1}{2}(A+I), \frac{1}{2}(A+2 I) ; T+\frac{1}{2} I ; \frac{-\xi^{2}}{v \lambda^{2}}\right) .
\end{aligned}
$$

Theorem 15. Let $\xi \in \mathbb{C}, v>0, \Re(\lambda)>0, m \in \mathbb{N}_{0}$. Also, let $T$ and $A$ be a positive stable matrices in $C^{d \times d}$ such that $T+n I$ is invertible for all $n \in \mathbb{N}_{0}$. The following Laplace transform formula hold

$$
\begin{aligned}
\mathscr{L}\left\{u^{A} \operatorname{HP}_{m}(T ; u, v): \lambda\right\} \\
=(4 v)^{m}\left(T+\frac{1}{2} I\right)_{m} \lambda^{-(A+I)} \Gamma(A+I)_{3} \mathbf{F}_{1} \\
\quad \cdot\left(-m I, \frac{1}{2}(A+I), \frac{1}{2}(A+2 I) ; T+\frac{1}{2} I ; \frac{-1}{v \lambda^{2}}\right) .
\end{aligned}
$$

The above Theorems lead to the following special cases.

Corollary 16. For $T$ is a positive stable matrix in $C^{d \times d}$, and $\mathfrak{R}(\lambda)>0$, then we have the following Laplace transforms

$$
\begin{aligned}
& \mathscr{L}\left\{u^{T-\frac{1}{2} I} \operatorname{HP}_{m}(T ; \xi \sqrt{u}, v): \lambda\right\} \\
& =(4 v)^{m} \lambda^{-\left(A+\frac{l}{2}\right)} \Gamma\left(A+\left(m+\frac{1}{2}\right) I\right)\left(1+\frac{\xi^{2}}{4 v \lambda}\right)^{m}, \\
& \mathscr{L}\left\{u^{2 T-I} \operatorname{HP}_{m}(T ; \xi u, v): \lambda\right\} \\
& =(4 v)^{m}\left(T+\frac{1}{2} I\right)_{m} \lambda^{-2 T} \Gamma(2 T)_{2} \mathbf{F}_{0}\left(-m I, T ;-; \frac{-\xi^{2}}{v \lambda^{2}}\right) \\
& \mathscr{L}\left\{u^{2 T-I} \mathbb{H P}_{m}(T ; u, v): \lambda\right\} \\
& \quad=(4 v)^{m}\left(T+\frac{1}{2} I\right)_{m} \lambda^{-(2 T)} \Gamma(2 T)_{3} \mathbf{F}_{1}\left(-m I, T ;-; \frac{-1}{v \lambda^{2}}\right) .
\end{aligned}
$$

\section{Fractional Calculus Approach}

Here, we consider the Riemann. Liouville fractional integral and derivative operators $\mathbf{I}^{\gamma}$ and $D_{z}^{\gamma}$ of order $\gamma \in C, \mathfrak{R}(\gamma)>0$, respectively (see, for details, [19])

$$
\left(\mathbf{I}^{\gamma} f(\tau)\right)(z)=\frac{1}{\Gamma(\gamma)} \int_{0}^{z} f(\tau)(z-\tau)^{\gamma-1} d \tau,
$$

where $f(\tau)$ is a function of $\tau$ and some square matrices so that this integral converges. 


$$
D_{z}^{\gamma}\{f(z)\}= \begin{cases}\frac{1}{\Gamma(-\gamma)} \int_{0}^{z}(z-\tau)^{-\gamma-1} f(\tau) d \tau, & (\mathfrak{R}(\gamma)<0), \\ \frac{d^{n}}{d z^{n}}\left(D_{z}^{\gamma-n}\{f(z)\}\right), & (n-1 \leq \mathfrak{R}(\gamma)<n(n \in N)),\end{cases}
$$

where

$$
D_{z}^{\gamma}\left\{z^{\beta}\right\}=\frac{\Gamma(\beta+I)}{\Gamma(\beta-(\gamma-1) I)} z^{\beta-\gamma} \mathfrak{R}(\beta)>-1
$$

It is noted in passing that (29) and (30) are applied in recent works, for example, (see [16-22]).

Theorem 17. For the generalized heat matrix polynomials of degree $m \in \mathbb{N}_{0}$, the following fractional integral operator holds true:

$$
\begin{aligned}
\mathbf{I}^{\gamma}\left\{\xi^{T-\frac{1}{2}} \mathbb{H P}_{m}(T ; \sqrt{\xi}, v)\right\}= & \Gamma\left(T+\left(m+\frac{1}{2}\right) I\right) \Gamma^{-1} \\
& \cdot\left(T+\left(\gamma+m+\frac{1}{2}\right) I\right) \\
& \times \xi^{T+\left(\gamma-\frac{1}{2}\right) I} \mathbb{H P}_{m}(T+\gamma I ; \sqrt{\xi}, v) .
\end{aligned}
$$

Proof. From (29) and (11), we have

$$
\begin{aligned}
\mathbf{I}^{\gamma}\left\{\xi^{T-\frac{1}{2} I} \mathbb{H P}_{m}(T ; \sqrt{\xi}, v)\right\}= & \frac{1}{\Gamma(\gamma)} \int_{0}^{\xi}(\xi-\tau)^{\gamma-1} \tau^{T-\frac{1}{2} I} \mathbb{H P}_{m} \\
& \cdot(T ; \sqrt{\tau}, v) d \tau .
\end{aligned}
$$

Setting $\tau=\xi \eta$ and after a simplification, we get

$$
\begin{aligned}
\mathbf{I}^{\gamma}\{ & \left.\xi^{T-\frac{1}{2} I} \mathbb{H P}_{m}(T ; \sqrt{\xi}, v)\right\} \\
= & \frac{\xi^{T+(\gamma-1 / 2) I}}{\Gamma(\gamma)} \int_{0}^{1} \eta^{T-\frac{1}{2} I}(1-\eta)^{\gamma-1} \mathbb{H P}_{m}(T ; \sqrt{\xi \eta}, v) d \eta \\
= & \xi^{T+\left(\gamma-\frac{1}{2}\right) I}(4 v)^{m}\left(T+\frac{1}{2} I\right)_{m} \sum_{s=0}^{m}(-m I)_{s}\left[\left(T+\frac{1}{2} I\right)_{s}\right]^{-1} \Gamma \\
& \cdot\left(T+\left(s+\frac{1}{2}\right) I\right) \times \Gamma^{-1}\left(T+\left(s+\gamma+\frac{1}{2}\right) I\right) \frac{\left(-(\sqrt{\xi})^{2} / 4 v\right)^{s}}{s !},
\end{aligned}
$$

whose last summation, in view of (11), is easily seen to arrive at the expression in (32). This completes the proof of Theorem 17.
Theorem 18. For the generalized heat matrix polynomials $\operatorname{HP}_{m}(T ; \xi, v)$, we have the following formula

$$
\begin{aligned}
& \sum_{m=0}^{\infty}\left[\left(T+\frac{1}{2} I\right)_{m}\right]_{m}^{-1} \frac{(\beta)_{m}}{(\gamma)_{m}} \mathbb{H P}_{m}(T ; \xi, v) \frac{t^{m}}{m !} \mathbf{F}_{1}(\gamma-\beta ; \gamma+m ; 4 v t) \\
& \quad=\exp (4 v t)_{1} \mathbf{F}_{1}\left(\beta I ; T+\frac{1}{2} I ; \xi^{2} t\right), \mathfrak{R}(\beta)>0, \Re(\gamma)>0 .
\end{aligned}
$$

Proof. According to (15), we find that

$$
\begin{aligned}
& \exp (-4 v t) \sum_{m=0}^{\infty}\left[\left(T+\frac{1}{2} I\right)_{m}\right]^{-1} \operatorname{HP}_{m}(T ; \xi, v) \frac{t^{m}}{m !} \\
& ={ }_{0} \mathbf{F}_{1}\left(-; T+\frac{1}{2} I ; \xi^{2} t\right) .
\end{aligned}
$$

Assume that the left-hand side of (35) be denoted by J. Multiply the left-hand side (35) by $t^{\beta-1}$ and applying the relation (31), we get

$$
\begin{aligned}
\mathrm{J}= & \frac{\Gamma(\beta)}{\Gamma(\gamma)} t^{\gamma-1} \sum_{m=0}^{\infty} \sum_{s=0}^{\infty}\left[\left(T+\frac{1}{2} I\right)_{m}\right]^{-1} \mathbb{H P}_{m} \\
& \cdot(T ; \xi, v)(4 v)^{s} \frac{(-1)^{s}(\beta)_{m+s} t^{m+s}}{(\gamma)_{m+s} s ! m !} \\
= & \frac{\Gamma(\beta)}{\Gamma(\gamma)} t^{\gamma-1} \sum_{m=0}^{\infty}\left[\left(T+\frac{1}{2} I\right)_{m}\right]^{-1} \mathbb{H P}_{m}(T ; \xi, v) \frac{(\beta)_{m} t^{m}}{(\gamma)_{m} m !} \mathbf{F}_{1} \\
& \cdot\left(\beta+m ; \gamma+\frac{1}{2} ;-4 v t\right) .
\end{aligned}
$$

Invoking Kummer's matrix transformation [5] leads to

$$
\begin{aligned}
\mathrm{J}= & \frac{\Gamma(\beta)}{\Gamma(\gamma)} t^{\gamma-1} \exp (-4 v t) \sum_{m=0}^{\infty}\left[\left(T+\frac{1}{2} I\right)_{m}\right]^{-1} \mathbb{H P}_{m} \\
& \cdot(T ; \xi, v) \frac{(\beta)_{m} t^{m}}{(\gamma)_{m} m !} \mathbf{F}_{1}(\gamma-\beta ; \gamma+m ; 4 v t) .
\end{aligned}
$$

It is easy to multiply right-hand side of (35) by $t^{\beta-1}$ and applying the fractional differentiation operator $D_{t}^{\beta-\gamma}$ from (31), whereupon this completes the establishment of the Theorem 18.

\section{Concluding Remarks}

In [23], Rosenbloom and Widder investigated expansions of solutions $u(x, t)$ of the heat equation $u_{x x}=u_{t}$ in series of polynomial solutions $v_{n}(x, t)$. Further, Haimo and Markett $[33,34]$ discussed the generalized heat equation

$$
\Delta_{x} u(x, t)=\frac{\partial}{\partial t} u(x, t)
$$

where $\Delta_{x} h(x)=h^{\prime \prime}(x)+(2 v / x) h^{\prime}(x), v$ is a fixed positive number and they introduced the generalized heat polynomial solution of (39) as 


$$
\mathbb{P}_{n, v}(x, t)=\sum_{j=0}^{n} 2^{2 j}\left(\begin{array}{l}
n \\
j
\end{array}\right) \frac{\Gamma(v+1 / 2+n)}{\Gamma(\nu+1 / 2+n-j)} x^{2 n-2 j} t^{j} .
$$

Note that $\mathbb{P}_{n, 0}(x, t)=v_{2 n}(x, t)$ is the ordinary heat polynomials of even order. Also, $\mathbb{P}_{n, 0}(x,-1)=H_{2 n}(x / 2)$ is the Hermite polynomials of even order.

Recently, many studies and extensions of the well-known special matrix polynomials have been in a focus of increasing attention leading to new and interesting problems. In this perspective, we defined the generalized heat matrix polynomials. Also, we have given some of their main properties, namely, the generating matrix functions, finite sum formulas, Laplace integral transforms, and fractional calculus operators. Further, This study is assumed to be a generalization of the scalar cases $[27,33,34]$ to the matrix setting. In addition, this approach allows to derive several new integral and differential representations that can be used in theoretical, applicable aspects like the boundary value problems and the numerical algorithms. Additional research and application on this topic is now under preparation and will be presented in forthcoming works.

\section{Data Availability}

No data were used to support the study.

\section{Conflicts of Interest}

This work does not have any conflicts of interest.

\section{Acknowledgments}

The first-named author extends their appreciation to the Deanship of Scientific Research at King Khalid University for funding work through research groups program under grant (R.G.P.1/15/42).

\section{References}

[1] G. Ariznabarreta, J. C. García-Ardila, M. Mañas, and F. Marcellán, "Matrix biorthogonal polynomials on the real line: Geronimus transformations," Bulletin of mathematical sciences, vol. 9, article 1950007., 2019.

[2] A. Bakhet and F. He, "On 2-variables Konhauser matrix polynomials and their fractional integrals," Mathematics, vol. 8, article 232, 2020.

[3] G. Heckman and M. van Pruijssen, "Matrix valued orthogonal polynomials for Gelfand pairs of rank one," Tohoku Mathematical Journal, vol. 68, pp. 407-436, 2016.

[4] A. Iserles and M. Webb, "A family of orthogonal rational functions and other orthogonal systems with a skew-Hermitian differentiation matrix," Journal of Fourier Analysis and Applications, vol. 26, no. 1, p. 19, 2020.

[5] R. Dwivedi and V. Sahai, "Lie algebras of matrix difference differential operators and special matrix functions," Advances in Applied Mathematics, vol. 122, pp. 102-109, 2021.

[6] R. Dwivedi and V. Sahai, "On q-special matrix functions using quantum algebraic techniques," Reports on Mathematical Physics, vol. 85, no. 2, pp. 253-265, 2020.
[7] M. Hidan, M. Akel, S. Boulaaras, and M. Abdalla, "On behavior Laplace integral operators with generalized Bessel matrix polynomials and related functions," Journal of Function Spaces, vol. 2021, Article ID 9967855, 11 pages, 2021.

[8] M. Abdalla, "On Hankel transforms of generalized Bessel matrix polynomials," AIMS Mathematics, vol. 6, no. 6, pp. 6122-6139, 2021.

[9] M. Abdalla, S. Boulaaras, and M. Akel, "On Fourier-Bessel matrix transforms and applications," Mathematical Methods in the Applied Sciences, 2021.

[10] L. Rodman, "Orthogonal matrix polynomials," in Orthogonal Polynomials: Theory and Practice, P. Nevai, Ed., vol. 294, pp. 345-362, NATO ASI Series (Mathematical and Physical Sciences); Springer, Berlin, Germany, 1990.

[11] M. Abdalla, "Special matrix functions: characteristics, achievements and future directions," Linear Multilinear Algebra, vol. 68, no. 1, pp. 1-28, 2020.

[12] P. Agarwal, D. Baleanu, Y. Chen, S. Momani, and J. Machado, Fractional Calculus: ICFDA 2018, Springer Proceedings in Mathematics Statistics 303 (Hardback), Amman, Jordan, 2020.

[13] M. K. Bansal, D. Kumar, K. S. Nisar, and J. Singh, "Certain fractional calculus and integral transform results of incomplete $\aleph$-functions with applications," Mathematical Methods in the Applied Sciences, vol. 43, no. 8, pp. 5602-5614, 2020.

[14] Y. Singh, V. Gill, J. Singh, D. Kumar, and I. Khan, "Computable generalization of fractional kinetic equation with special functions," Journal of King Saud University-Science, vol. 33, no. 1, p. 101221, 2021.

[15] M. K. Bansal, S. Lal, D. Kumar, S. Kumar, and J. Singh, "Fractional differential equation pertaining to an integral operator involving incompleteH-function in the kernel," Mathematical Methods in the Applied Sciences, pp. 1-12, 2020.

[16] M. Abdalla, M. Akel, and J. Choi, "Certain matrix RiemannLiouville fractional integrals associated with functions involving generalized Bessel matrix polynomials," Symmetry, vol. 13 , no. 4 , p. $622,2021$.

[17] M. Abdalla and M. Hidan, "Fractional orders of the generalized Bessel matrix polynomials," European Journal of Pure and Applied Mathematics, vol. 10, pp. 995-1004, 2017.

[18] M. Abdalla, "Fractional operators for the Wright hypergeometric matrix functions," Advances in Difference Equations, vol. 2020, no. 1, 2020.

[19] A. M. Mathai and H. J. Haubold, An Introduction to Fractional Calculus, Nova Science Publishers, NY, USA, 2017.

[20] A. Bakhet, Y. Jiao, and F. He, "On the Wright hypergeometric matrix functions and their fractional calculus," Integral Transforms and Special Functions, vol. 30, no. 2, pp. 138-156, 2019.

[21] M. Zayed, M. Abul-Ez, M. Abdalla, and N. Saad, "On the fractional order Rodrigues formula for the shifted Legendre-type matrix polynomials," Mathematics, vol. 8, p. 136, 2020.

[22] M. Zayed, M. Hidan, M. Abdalla, and M. Abul-Ez, "Fractional order of Legendre-type matrix polynomials," Advances in Difference Equations, vol. 2020, 13 pages, 2020.

[23] P. C. Rosenbloom and D. V. Widder, "Expansions in terms of heat polynomials and associated functions," Transactions of the American Mathematical Society, vol. 92, no. 2, pp. 220266, 1959.

[24] L. R. Bragg, "The radial heat polynomials and related functions," Transactions of the American Mathematical Society, vol. 119, no. 2, pp. 270-290, 1965. 
[25] G. N. Hile and A. Stanoyevitch, "Heat polynomial analogs for higher order evolution equations," Electronic Journal of Differential Equations (EJDE), vol. 28, pp. 1-19, 2001.

[26] L. Shy-Der Lin, T. Shih-Tong Tu, and H. Srivastava, "Some generating function involving the stirling numbers of second kind," Rendiconti del Seminario Matematico, vol. 59, 2001.

[27] S. N. Kharin, "Special functions and heat polynomials for the solution of free boundary problems," AIP Conference Proceedings, vol. 1997, article 020047, 2018.

[28] B. Gürbüz and M. Sezer, "A new computational method based on Laguerre polynomials for solving certain nonlinear partial integro differential equations," Acta Physica Polonica A, vol. 132, no. 3, pp. 561-563, 2017.

[29] M. Gülsu, B. Gürbüz, Y. Öztürk, and M. Sezer, "Laguerre polynomial approach for solving linear delay difference equations," Applied Mathematics and Computation, vol. 217, pp. 67656776, 2001.

[30] B. Gürbüz and M. Sezer, "Laguerre polynomial solutions of a class of initial and boundary value problems arising in science and engineering fields," Acta Physica Polonica A, vol. 130, no. 1, pp. 194-197, 2016.

[31] J. Singh, D. Kumar, and M. K. Bansal, "Solution of nonlinear differential equation and special functions," Mathematical Methods in the Applied Sciences, vol. 43, no. 5, pp. 21062116, 2020.

[32] S. M. Boulaaras, A. Choucha, A. Zara, M. Abdalla, and B. Cheri, "Global existence and decay estimates of energy of solutions for a new class of p-Laplacian heat equations with logarithmic nonlinearity," Journal of Function Spaces, vol. 2021, Article ID 5558818, 11 pages, 2021.

[33] D. T. Haimo and C. Markett, "A representation theory for solutions of a higher order heat equation, I," Journal of Mathematical Analysis and Applications, vol. 168, no. 1, pp. 89-107, 1992.

[34] D. T. Haimo and C. Markett, "A representation theory for solutions of a higher order heat equation, II," Journal of Mathematical Analysis and Applications, vol. 168, no. 2, pp. 289305, 1992.

[35] G. N. Hile and A. Stanoyevitch, "Expansions of solutions of higher order evolution equations in series of generalized heat polynomials," Electronic Journal of Differential Equations, vol. 64, pp. 1-25, 2002.

[36] R. Horn and C. Johnson, Topics in Matrix Analysis, Cambridge University Press, Cambridge, 2011.

[37] N. J. Higham, Functions of Matrices Theory and Computation, Siam, USA, 2008.

[38] L. Jódar and J. C. Cortés, "Some properties of gamma and beta matrix functions," Applied Mathematics Letters, vol. 11, no. 1, pp. 89-93, 1998.

[39] L. Jódar and J. C. Cortés, "On the hypergeometric matrix function," Journal of Computational and Applied Mathematics, vol. 99, no. 1-2, pp. 205-217, 1998.

[40] J. Sastre, E. Defez, and L. Jodar, “Application of Laguerre matrix polynomials to the numerical inversion of Laplace transforms of matrix functions," Applied Mathematics Letters, vol. 24, no. 9, pp. 1527-1532, 2011.

[41] B. Çekim and A. Altin, "Matrix analogues of some properties for Bessel functions," Journal of Mathematical Sciences, vol. 22, pp. 519-530, 2015. 